sich die Aktivität eines $\mathrm{Mg}$-abhängigen Enzyms mit dem Logarithmus der $\mathrm{Mg}$-Ionenkonzentration ändert. Aus diesen Gründen müßte die intrazelluläre $\mathrm{Mg}$-Ionenkonzentration schon erheblich ansteigen (oder abfallen), um die Aktivität $\mathrm{Mg}$-abhängiger Enzyme zu ändern. Die von Kochetov (2) beschriebene Aktivierung der Fructose-Diphosphatase (FDP-ase)-Aktivität in verdünnten Leberhomogenaten nach Zugabe von $\mathrm{Mg}$ läßt sich damit erklären, daß dieses Enzym zu seiner Aktivierung eine $\mathrm{Mg}$-Konzentration benötigt, die im verdünnten Homogenat ohne $\mathrm{Mg}$-Zusatz nicht vorhanden ist. Eine erhöhte Spaltung von Fructosediphosphat (FDP) nach Bestrahlung braucht aber keine $\mathrm{Mg}$-bedingte Aktivierung der FDP-ase zu sein. FDPase wird als allosterisches Enzym durch verschiedene Effektoren (AMP, FDP) beeinflußt (13), deren Verhalten in der Leber nach Ganzkörperbestrahlung nicht bekannt ist. Außerdem kann auch eine Freisetzung unspezifischer lysosomaler Phosphatase, die ebenfalls FDP spaltet, beteiligt sein. Eine derartige Freisetzung lysosomaler Phosphatase nach Einwirkung ionisierender Strahlen konnten wir in früheren Versuchen nachweisen (14).

Die von BACQ und Mitarbeitern (1) erwogene Erklärung der durch Fluoracetat erhöhten Überlebensdauer nach letaler Bestrahlung durch Herabsetzung der $\mathrm{Mg}$-Ionenkonzentration in der Zelle zum Zeitpunkt der Strahleneinwirkung ist unwahrscheinlich, da eine wirksame Abnahme der Mg-Ionenkonzentration unter Fluoracetat nicht anzunehmen ist und Fluoracetat auch über andere Mechanismen wirken könnte. Diese Möglichkeit wurde experimentell nicht überprüft, da der $\mathrm{Mg}$-Gehalt tierischer Zellen durch $\mathrm{Mg}$-freie Diät nur bei wachsenden Tieren und nur relativ geringfügig herabgesetzt werden kann. Wenn aber bei Wachstum unter $\mathrm{Mg}$-Mangelbedingungen der $\mathrm{Mg}$-Gehalt ( $z$. B. bei Bakterien) vermindert wird, treten komplexe Änderungen des Zellstoffwechsels ein (15), die keinen Rückschluß auf eine kausale Beziehung zwischen der Strahlenwirkung und dem $\mathrm{Mg}$-Mangel erlauben.

\title{
Literatur
}

1. BACQ, Z. M., S. LrébecQ-HutTer und C. LiébeCQ, Radiation Res. 13, 286 (1960). - 2. Kochetov, G. A., Biochim., Moskva 26, 287 (1961). - 3. MacIntyre, I. und D. Davidsson, Biochem. J. 70, 456 (1958). - 4. Günther, Th. und Ch. Alter, diese Z. 5, 67 (1967). - 5. Orange, M. und H. C. Rhein, J. biol. Chemistry 189, 379 (1951). - 6. Breuer, H. und H. K. Parchwitz, Z. Naturforsch. 156, 671 (1960). - 7. HAGEN, U., in: Ergebnisse der medizinischen Strahlenforschung S. 487-527, Hrsg. H. R. Schinz, R. Glauner und A. Rürtimann; G. Thieme, Stuttgart (1964). 8. Rogers, T. A. und P. E. Mahan, Proc. Soc. Exper. Biol. Med.,
New York 100, 235 (1959). - 9. Breuer, H., H. K. Parchwitz und C. Winkrer, Strahlentherapie 105, 579 (1958). - 10. DuCKWORTH, J., W. GODdEN und G. M. Warnock, Biochem. J. 34, 97 (1940). - 11. Jackson, K. L., R. Rhodes und C. Entenman, Radiation Res. 8, 361 (1958). - 12. GüNTHER, TH., Z. Naturforsch. 22b, 149 (1967). - 13. Underwood, A. H. und E. A. Newsholme, Biochem. J. 95, 767 (1965). - 14. Bublitz, G., H. J. Merker und Th. GüNTHER, Fortschr. Röntgenstr. 108, 238, (1968). - 15. GÜNThER, Th. und P. Mariss, Hoppe-Seyler's Z. physiol. Chem. (im Druck).
Priv.-Doz. Dr. Th. Günther 1 Berlin 33,

Arnimallee 22

\section{Die Inaktivierung von Östradiol durch Oxydation nach Leberschädigung bei Ratten und bei leberkranken Patienten}

\author{
Untersucbungen mit Stoffivechsel-labil markiertem Östradiol- [17 $\alpha-T]$
}

Von M. Wenzel, K. U. Blum und E. KraAs

\author{
Aus dem Physiologisch-Chemischen Institut (Abt.: Prof. Dr. Dr. E. Schütte) \\ und der I. Medizinischen Klinik der Freien Universität Beriin (Direktor: Prof. Dr. Dr. b. c. Frbr. v. Kress)
}

(Eingegangen am 1. März 1968)

Herrn Prof. Dr. Dr. Ernst Schütte zum 60. Geburtstag geividmet

Mit Hilfe von Östradiol-[17 $\alpha-T]$ wird die in-vivo-Oxydation von Östradiol zu Östron durch die Bestimmung des Tritiumgehaltes des Körperwassers bei gesunden und lebergeschädigten Ratten sowie bei leberkranken Patienten gemessen.

Entgegen der bisher geläufigen Meinung findet man bei chronisch leberkranken Patienten und bei Ratten nach chronischer Leberschädigung durch Thioacetamid eine gesteigerte Inaktivierung des Ostradiols durch Oxydation zu Óstron.

Akute Leberschädigung bei Ratten durch Gabe von Tetrachlorkohlenstoff führt dagegen zu einer verminderten Östradiol-Oxydation.

The in vivo oxidation of oestradiol to oestrone was measured in healthy and liver-damaged rats and in patients with illnesses of the liver by the determination of the tritium concentration in the body water, following the administration of [17 $\alpha$-T]-oestradiol.

Contrary to the current view, the inactivation of oestradiol by oxidation to oestrone is increased in patients with chronic liver disease and in rats after chronic liver damage with thioacetamide.

Acute liver damage in tats, caused by carbon tetrachloride, results, however, in a decreased oxidation of oestradiol. 
Da bei der Inaktivierung von Steroiden die Leber die entscheidende Rolle spielt, kann bei Schädigungen des Leberparenchyms der Abbau der Steroid-Hormone gestört werden $(1,2)$. So führen chronische Erkrankungen des Leberparenchyms oft zu Bildern, die mit einer verstärkten Östrogenwirkung erklärt werden. Es war daher naheliegend, diese klinische Erscheinung durch einen verminderten Östradiol-Abbau zu erklären. $\mathrm{Da}$ Östradiol sowohl durch Oxydation am C-17-Atom als auch durch Hydroxylierung und Ester-Bildung mit Glucuronsäure und Schwefelsäure in biologisch weniger aktive Derivate überführt werden kann, ist eine verminderte Östradiol-Inaktivierung durch die Störung verschiedener Reaktionen möglich. Inwieweit Leberschädigungen zu Änderungen des oxydativen Abbau des Östradiols am C-17-Atom führen können, soll die folgende Arbeit klären.

Das Studium gerade dieser Reaktion ist deshalb für unsere Frage wichtig, weil die Oxydation von Östradiol $z u$ Östron zu einer Verminderung der östrogenen Wirkung des Steroid-Moleküls um den Faktor 10 führt. Die Untersuchungen wurden mit Hilfe von „Stoffwechsel-labil"c-markiertem Östradiol-[17 $\alpha$-T] durchgeführt. Damit gelingt es auf einfache Weise, innerhalb des lebenden Organismus spezifisch die Mindestoxydation von Östradiol zu Östron $(3,4)$ oder die anderer Hydroxy-Steroide $(5,6) \mathrm{zu}$ den korrespondierenden Steroid-Ketonen zu erfassen.

\section{Material und Methoden}

Für die Versuche verwendeten wir geschlechtsreife männliche Tiere eines hauseigenen Wistar-Rattenstammes. Die Tiere waren 4 bis 6 Monate alt und hatten ein Gewicht zwischen 140 und $210 \mathrm{~g}$. Sie erhielten Futter (,Altromin") und Wasser ad libitum.

\section{Vorbehandlung der Tiere}

Wir versuchten mit Tetrachlorkohlenstoff und Åthionin eine akute Lebervergiftung zu erreichen und mit Thioacetamid eine chronische Leberschädigung nachzuahmen.

\section{Mit Tetracblorkoblenstoff}

Untersucht wurden insgesamt 8 Ratten, von denen jeweils 2 Tiere über vier Tage verteilt bestimmte Mengen Tetrachlorkohlenstoff s. c. unter die Rückenhaut erhielten. Das erste Rattenpaar erhielt insgesamt $3,6 \mathrm{ml} / \mathrm{kg}$, das zweite insgesamt $4,3 \mathrm{ml} / \mathrm{kg}$, das dritte und vierte insgesamt $19,4 \mathrm{bzw} .29,4 \mathrm{~m} / \mathrm{kg}$. Die so vorbehandelten Tiere wurden am fünften Tag untersucht.

\section{Mit Atbionin}

Die zweite Gruppe bestand aus 4 Tieren, von denen jeweils zwei Ratten über vier Tage verteilt bestimmte Äthioninmengen i. p. injiziert bekamen. Das erste Paar erhielt insgesamt $1,5 \mathrm{~g}$ Äthionin $/ \mathrm{kg}$, das zweite Paar 2,1 g Äthionin/kg.

\section{Mit Tbioacetamid}

Untersucht wurden drei Ratten, denen über 100 Tage lang jeweils $30 \mathrm{mg}$ Thioacetamid pro Tag und Tier dem Futter beigemischt worden war. Diese Tiere waren die letzten drei einer Gruppe von 12 Tieren; die anderen 9 Tiere starben vor dem hundertsten Tag.

Radioaktive Substanz und Versuchsanordnung zur Gewinnung des Atemwassers

Die Herstellung des Östradiol-[17 $\alpha-T]$ wurde früher beschrieben

(3). Die Tiere erhielten am Versuchstag jeweils $0,25 \mathrm{mg} / \mathrm{kg}$
Östradiol-[17 $\alpha$-T] (etwa $25 \mu \mathrm{C}$ ) in $0,5 \mathrm{ml}$ Propylenglykol gelöst i. $m$. injiziert. Anschließend wurden die Ratten in einen speziellen Käfig $^{1}$ ) gesetzt, dem getrocknete Luft zugeführt wird. Der von den Tieren an die Ausatmungsluft abgegebene Wasserdampf wird in einer Kühlfalle kondensiert. Die spezifische Aktivität der Wasserproben wurde im „Tri-Carb“ Modell 314 Ex der Firma Packard gemessen.

Berechnung der Steroid-Oxydation

Die Steroid-Oxydation wird nach folgender Formel berechnet:

$$
\frac{\mu \mathrm{C} \text { pro } \mathrm{m} l \text { Körperwasser-Destillat }}{\mu \mathrm{C}_{\text {inj. Ostr. pro } \mathrm{m} l \text { Körperwasșer }}} \cdot 100=\underset{\text { des Ostradiols }}{\%} \text { Mindestoxydation }
$$

Der zugrunde liegende Reaktionnsablauf läßt sich folgendermaßen zusammenfassen:

$$
\begin{gathered}
\text { Ostradiol-[17 } \alpha-\mathrm{T}]+\mathrm{NAD} \frac{\text { Steroid }}{\text { Dehydrogense }} \text { Ostron } \\
+ \text { NAD-T } \stackrel{\text { Atmungs- }}{\text { kette }} \text { HTO }
\end{gathered}
$$

Histologische Untersuchung

Die Lebern der Versuchstiere wurden histologisch aufgearbeitet (Fixierung in 10proz. Formalin, Gefrierschnittechnik, Färbung nach vaN Greson). Bei der Untersuchung war uns Priv.-Doz. Dr. LINDLAR, Pathologisches Institut der Freien Universität Berlin behilflich, wofür wir ihm vielmals danken.

Versuchsanordnung bei Patienten

Die Patienten erhielten $148 \mu \mathrm{g}$ Östradiol-[17 $\alpha-T]$ mit einer Aktivität von $99 \mu \mathrm{C}$ in Propylenglykol/0,9proz. NaCl-Lösung (1:1) gelöst i. m. injiziert. Bei dieser Dosis hält sich einerseits die Hormonmenge im physiologischen Bereich, andererseits liegt die Radioaktivität unterhalb der gesetzlichen Freigrenze (7).

Versuchspersonen

Die Diagnosen der leberkranken Patienten sind in der Reihenfolge

\begin{tabular}{|c|c|c|c|}
\hline \multirow[b]{2}{*}{ G. P. $0^{n}$} & \multirow[b]{2}{*}{72 Jahre } & \multirow[b]{2}{*}{$60,4 \mathrm{~kg}$} & \multirow{4}{*}{$\begin{array}{l}\text { Chronische Hepatitis mit } \\
\text { Obergang in Leberzirrhose. } \\
\text { Histologisch: Chronische, } \\
\text { interstitielle Hepatitis mit } \\
\text { möglichem Ubergang in } \\
\text { Leberzirrhose. } \\
\text { Leberzirrhose, dekompen- } \\
\text { sierter Hypertonus. } \\
\text { Histologisch: Atrophische } \\
\text { Leberzirrhose. } \\
\text { Hepatitis epidemica. } \\
\text { Histologisch: Mittelschwere, }\end{array}$} \\
\hline & & & \\
\hline H. C. $\sigma^{\prime \prime}$ & 73 Jahre & $63,9 \mathrm{~kg}$ & \\
\hline C. St. $ᄋ$ & 88 Jahre & $53,3 \mathrm{~kg}$ & \\
\hline K. F. O & - & $78,5 \mathrm{~kg}$ & $\begin{array}{l}\text { Leberzirrhose. } \\
\text { Histologisch: Leberzirrhose } \\
\text { mit dystrophischem Schub. }\end{array}$ \\
\hline R. K. $ᄋ$ & 61 Jahre & $64,5 \mathrm{~kg}$ & $\begin{array}{l}\text { Infektiöse Hepatitis. } \\
\text { Histologisch: Interstitielle, } \\
\text { chronische Hepatitis. }\end{array}$ \\
\hline $\begin{array}{l}\text { O. P. }{ }^{*} \\
\text { E. K. }\end{array}$ & $\begin{array}{l}60 \text { Jahre } \\
7.6 \text { Jahre }\end{array}$ & $\begin{array}{l}84,0 \mathrm{~kg} \\
51,4 \mathrm{~kg}\end{array}$ & $\begin{array}{l}\text { Leberzirrhose. } \\
\text { Leberzirrhose. }\end{array}$ \\
\hline
\end{tabular}
(von oben nach unten, zur Zeit 4 Stdn. nach Injektion) der Kurven der Abbildung 2 aufgeführt:

\section{Ergebnisse}

\section{Östradioloxydation bei Ratten}

Abbildung 1 zeigt die Zeițabhängigkeit der Mindestoxydation von Östradiol- $[17 \alpha-T]$ zu OOstron bei gesunden männlichen Ratten und bèi Tieren nach verschiedenen Formen der Leberschädigung. Danach ergibt sich bei den gesunden Tieren bis zu 9 Stdn. nach der Injektion ein langsames Ansteigen der HTO-Konzentration im Körperwasser und daher der Werte für die ÖstradiolOxydation. Innerhalb von $9 \mathrm{Stdn}$. sind $66 \pm 6 \%$ der injizierten Dosis oxydiert. Danach fällt der HTO-

1) Hersteller: Fa. Witels, Albert \& Co., 1 Berlin 42, Ullsteinstr. 227 
Spiegel im Körperwasser der Tiere - wie in einem gesonderten Versuch gezeigt werden konnte - mit einer Halbwertszeit von 4,3 Tagen ab, was mit Messungen von THompson (8) über die biologische Halbwertszeit von HTO bei Ratten übereinstimmt. Es wird also nach 9 Stdn. kein weiteres Östradiol mehr oxydiert.

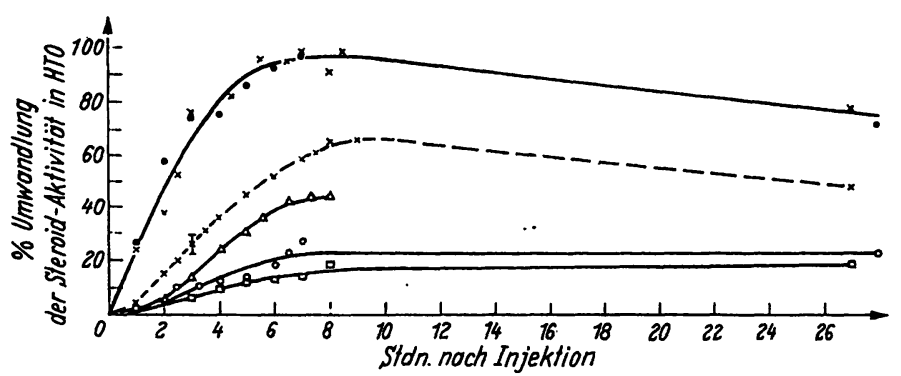

Abb. 1

Zeitabhängigkeit der Ostradiol-Oxydation am C-17 bei gesunden Ratten und nach Leberschädigung
Die Kontrollkurve wurde nach den Mittelwerten von insgesamt 12 Tieren eingezeichnet. Der mittlere Fehler des Mittelwertes ist als Zur Versuchsdurchführung vgl. Methodik $0 \times-x$ Thioacetamid $30 \mathrm{mg} /$ Ratte 100 Tage $\Delta-\Delta 4,3 \mathrm{ml}, \mathrm{O}-\mathrm{O} \times 19,2 \mathrm{ml}$, $\square-\square 29,4 \mathrm{ml} \mathrm{CCl} / \mathrm{kg}$ Ratte

Im Gegensatz hierzu ist die Oxydationsrate bei den Tetrachlorkohlenstoff-vergifteten Tieren niedriger. Innerhalb von 8 Stdn. sind bei den mit $4,3 \mathrm{ml}$ Tetrachlorkohlenstoff $/ \mathrm{kg}$ vorbehandelten Tieren etwa $40 \%$ der injizierten Dosis, bei den Tieren mit 29,4 ml Tetrachlorkohlenstoff/kg Vorbehandlung sogar nur $17 \%$ des injizierten Steroids oxydiert. Es wird also bei derartig starker Leberschädigung nur noch $1 / 4$ der ÖstradiolMenge oxydiert, die die Kontrolltiere in der gleichen Zeit abbauen.

Der besseren Ubersichtlichkeit wegen wurde in Abbildung 1 datauf vetzichtet, auch die Meßergebnisse der Tiergruppen mit $3,6 \mathrm{~m} l$ Tetrachlorkohlenstoff $/ \mathrm{kg}$ einzutragen. Die Exgebnisse zeigen dieselbe Tendenz und fügen sich gut in die abgebildeten Kurven ein.

Im Gegensatz hierzu steigt bei Rattenmännchen nach chronischer Vergiftung mit Thioacetamid die HTOKonzentration im Körperwasser und daher die Östradiol-Oxydation wesentlich schneller an als bei den Kontrollen. Innerhalb von 7 Stdn. wird etwa $96 \%$ der injizierten Dosis oxydiert. Die mit Thioacetamid vorbehandelten Tiere oxydieren also das injizierte Östradiol-[17 $[\alpha-T]$ doppelt so schnell wie die Kontrollen, und haben nach 7 Stdn. das gesamte injizierte Östradiol zu 17-Ketoverbindungen umgesetzt.

\section{Histologie .}

Um eine morphologische Kontrolle der Lebervergiftungen zu haben, wurden die Lebern der Versuchstiere histologisch untersucht.

Tetrachlorkohlenstoffvergiftung führt in der Leber zu überwiegend großtropfiger starker Verfettung besonders zentral und in der Intermediärzone sowie in den Kupprerschen Sternzellen. Das Parenchym in der Läppchenperipherie ist weitgehend normal. Man sieht vielfach verdämmernde Kerne und Thrombogenisierung des Cytoplasmas. Das Bindegewebe ist nicht vermehrt. Im Vergleich hierzu fällt bei Thioacetamid-Vergiftung (nach 100 Tagen) besonders die erhebliche, feinnetzige, periportale
Fibrose mit deutlichem Läppchenumbau und dem Erscheinen von Regeneratknoten auf.

Bei Untersuchungen an mit Äthionin vorbehandelten Tieren zeigte sich keine merkliche Abweichung von den Kontrolltieren.

Untersuchungen über die Östradioloxydation beim Menschen

Abbildung 2 zeigt die Abhängigkeit der Mindestoxydation von Östradiol-[17 $\alpha$-T] zu Östron bei lebergesunden und leberkranken Patienten. Dabei ergibt sich für die Östradiol-Oxydation, gemessen durch Bestimmung der HTO-Konzentration im Körperwasser (hier Gefrierdestillat des Urins) eine erstaunliche Parallelität zu den Ergebnissen bei den Ratten. Bei den

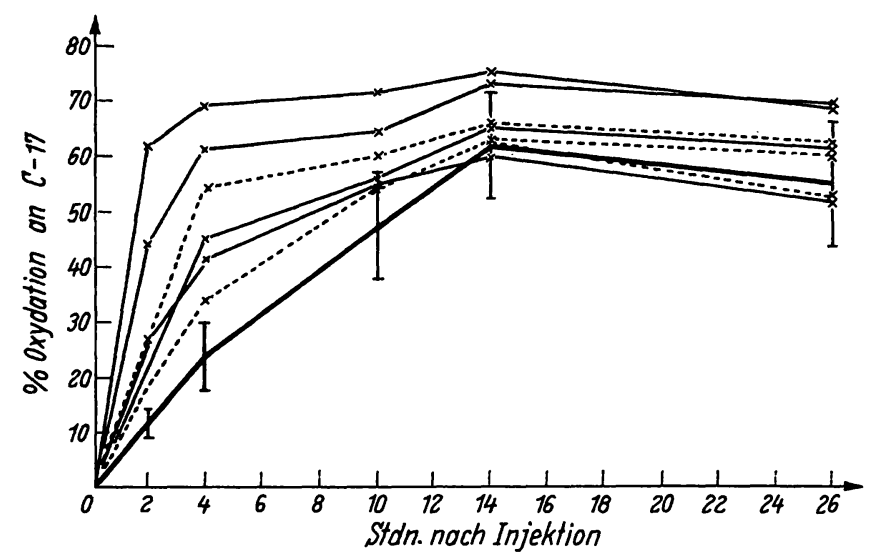

Abb. 2

Zeitabhängigkeit der Ostradiol-Oxydation an C-17 bei lebergesunden und leberkranken Versuchspersonen

Die Werte von lebergesunden Versuchspersonen (vgl. auch (7)) sind zu einer einer Kurve zusammengefaßt und der mittlere Fehler der

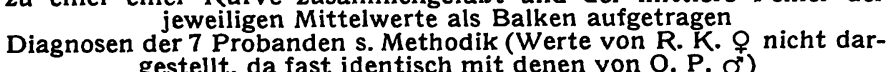

gestellt, da fast identisch mit denen von $\left.O . P . O^{\prime}\right)$
$\times-\times$ Gesunde, $\times \cdots \times$ leberkranke Frauen, $\times-\times$ leberkranke Männer

lebergesunden Personen ist innerhalb von $14 \mathrm{Stdn}$. der höchste Wert der Östradiol-Oxydation mit $62 \pm 10 \%$ der injizierten Dosis erreicht. Der anschließende Abfall des HTO-Spiegels im Körperwasser entspricht der Halbwertszeit von Tritiumwasser beim Menschen.

Bei chronisch Leberkranken findet sich eine beschleunigte Oxydation des Östradiols, so daß die HTOKonzentration im Körperwasser schneller ansteigt als bei den Lebergesunden. Während bei diesen nach 4 Stdn. im Durchschnitt erst $23 \pm 7 \%$ des Östradiols oxydiert worden sind, findet man zu diesem Zeitpunkt bei Leberkranken Werte, die einer 30-70proz. Oxydation von Östradiol entsprechen. Der höchste Wert der Östradiol-Oxydation liegt bei den leberkranken Patienten jedoch nicht wesentlich höher als bei Lebergesunden.

\section{Diskussion}

Nach den Ergebnissen der Tierversuche oxydieren gesunde und lebergeschädigte männliche Ratten Östradiol mit unterschiedlicher Geschwindigkeit zu Östron. So führt akute Leberschädigung mit Ausbildung einer Fettleber durch Tetrachlorkohlenstoffvergiftung zu einer Verminderung der Östradiol-Oxydation. Das stimmt mit den Befunden älterer Arbeiten [Übersicht bei 
(2)] überein, wonach Tetrachlorkohlenstoffgabe bei Ratten zu einer verminderten Östradiol-Inaktivierung führt. Bei der Inkubation von Östradiol mit Leberschnitten von Ratten, die mit p-Dimethylamino-azo-benzol behandelt und deren Lebern zirrhotisch geworden waren, zeigte sich ebenfalls eine verminderte Östradiol-Oxydation zu Östron (9).

Gleichfalls findet man bei Ratten, deren Lebern durch langdauernde Gabe von Diäthylnitrosamin geschädigt worden waren und bei denen sich Hepatome ausgebildet hatten, eine verminderte in-vivo-Oxydation von Östradiol (neben fehlendem Wasserstofftransport von Östradiol auf Androgene) (10).

Chronische Leberschädigung durch langdauerndes Behandeln mit Thioacetamid (mit dem histologischen Bild einer Leberzirrhose) führt dagegen zu einer erheblich beschleunigten Oxydation von Östradiol am C-17-Atom.

Analog den Ergebnissen nach chronischer Leberschädigung im Tierversuch zeigt sich bei chronisch leberkranken Patienten eine beschleunigte ÖstradiolOxydation gegenüber lebergesunden Personen. Bisher kann jedoch bei diesen Patienten keine Beziehung zwischen dem klinischen Krankheitsbild und den entsprechenden Laborbefunden (Elektrophorese, Bilirubin; Bromthalein-Exkretion, Enzymaktivitäten im Serum) einerseits und der Geschwindigkeit der ÖstradiolOxydation andererseits aufgestellt werden.

Untersuchungen an Patienten mit akuten Lebererkrankungen, die unter Umständen in Beziehung zur Tetrachlorkohlenstoffvergiftung der Ratte gestellt werden können, liegen bisher noch nicht vor, sind jedoch vorgesehen.

Die Gleichartigkeit der Ergebnisse des Tierversuches bei chronischer Leberschädigung und der Untersuchungen an Patienten mit chronischer Leberschädigung läßt jedoch die Anschauung als fraglich erscheinen, wonach diese Leberschäden zu einer verminderten Östradiol-Inaktivierung durch Oxydation führen und dadurch das klinische Bild des Hyperöstrogenismus bedingen. Da eine Störung der Östrogen-Konjugation bei Leberschädigung nicht nachzuweisen ist (11) und die Oxy- dation des Östradiols eher beschleunigt und vermehrt ist, erscheint die in der Klinik geläufige Anschauung der „verminderten Östradiol-Inaktivierung “ biochemisch nicht bewiesen.

Die Ansicht, daß die bei Männetn eintretenden Feminisierungserscheinungen bei Leberzirrhose eher durch einen Hypoandrogenismus als durch einen Hyperöstrogenismus zu erklären sind (12), erfährt durch diese Untersuchungen eine Unterstützung.

Worauf die veränderte Östradiol-Oxydation nach Leberschädigung zurückzuführen ist, läßt iich gegenwärtig nicht mit Sicherheit sagen.

Bei den Thioacetamid-geschädigten Ratten mag die beschleunigte Östradiol-Oxydation auf die Abmagerung der Tiere (Ausgangsgewicht 180-210 g; Gewicht nach Ende der Thioacetamid-Behandlung 110-130 g) zurückzuführen sein. Mangel an Fettgewebe könnte zu einer verminderten Speicherung des nicht-oxydierten Steroids (6) und damit einem beschleunigten Transport des Steroids zur Leber führen, in der die Oxydation stattfindet. $\mathrm{Daß}$ die Kapazität zur Steroid=Oxydation bei Ratten durch die hier verwendete Steroid-Dosis von $0,25 \mathrm{mg}$ Östradiol $/ \mathrm{kg}$ bei weitem nicht erschöpft ist, konnten wir früher zeigen (4).

An den oben erwähnten Zusammenhang wird man auch denken müssen, wenn man die beschleunigte Östradiol-Oxydation bei chronisch leberkranken $\mathrm{Pa}$ tienten erklären will, falls die betreffenden Patienten ein erhebliches Untergewicht haben.

Bei der akuten Leberschädigung bei Ratten durch Tetrachlorkohlenstoff schützt möglicherweise die Fetteinlagerung in das Lebergewebe das in die Leber transportierte Östradiol vor dem Zugriff der $17 \beta-$ Steroid-Dehydrogenase.

Inwieweit eine eventuelle Veränderung der Durchblutungsverhältnisse bei Leberschädigung (13) zu einer unterschiedlichen Östradiol-Oxydation in vivo führt, muß dahingestellt bleiben.

Für unermüdliche und gewissenhafte Mitarbeit danken wir Fräulein Barbara HaNisch.

Ein Teil der Arbeiten wurde durch die Deutsche Forschungsgemeinschaft gefördert.

\section{Literatur}

1. Kaiser, E., W. Rindt und G. Oertel, Acta hepato-splenologica 12, 12 (1965). - 2. Diczfalusy, E. und Ch. Lauritzen, Östrogene beim Menschen, Springer, Berlin-Göttingen-Heidelberg (1961). - 3. Wenzel, M., H. KLeUKer und P. E. Schulze, Z. Naturforsch. $21 b, 1178$ (1966). - 4. WenzeL, M. und E. KraAs, Acta endocr. K'hrn 56, 385 (1967). - 5. WenzeL, M. und I. BANGert, Klin. Wschr. 45, 1160 (1967). - 6. WENZEL, M., L. Pitzel und P. E. Schulze, Angew. Chemie 80, 191 (1968). -
7. Wenzel, M. und K. U. Blum, diese Z. 5, 202 (1967). - 8. Thompson, R. C., J. biol. Chemistry 200, 731 (1953). - 9. RYaN, K. und L. Engex, Endocrinology 52, 277 (1953). - 10. WeNZEL, M. und K. Pollow, Experientia, Basel (im Druck). - 11. BECK, K., H. A. KüHN und B. Nourare, Acta hepato-splenologica 14, 280 (1967). - 12. Szavas, F., C. Schirren und K. Becker, Acta hepato-splenologica 13, 356 (1966). 13. Preedy, J. und E. Arțkn, J. Clin. Invest. 35, 430 (1956).
Priv.-Doz. Dr. M. Wenzel 1000 Berlin 33

Königin-Luise-Str. 2-4 